\title{
Some thoughts about the IAU Strategic Plan in Latin America and the Caribbean
}

\author{
Silvia Torres-Peimbert
}

Instituto de Astronomía, Universidad Nacional Autónoma de México

\begin{abstract}
The distribution of overall education and culture among the population in Latin America and the Caribbean has a very wide range; this is also the case for astronomical engagement. The route to follow in the strategic plan needs to address this situation, for the different levels of development. In particular, guidelines should be established for the regional node(s) where achievable goals should be set up and evaluated periodically. I present a set of ideas on this subject.
\end{abstract}

The long-term vision of the IAU states (a) that all countries will participate at some level in international astronomical research, and (b) that all children throughout the world will be exposed to some knowledge about astronomy and the Universe at school in support of their education. Three aspects that need attention: (i) astronomy for the public, (ii) astronomy education, and (iii) astronomy at universities and research level.

I review some of the activities that have taken place in the continent. At the professional level, 13 Latin American Regional Meetings have been held since 1977; they have been by Argentina (2), Brasil (2), Chile (3) Mexico (3) Uruguay (1) and Venezuela (2); the next one will take place in Brasil. Regarding short courses at university level over the years, several International Schools for Young Astronomers have been held; I can mention: Trinidad Tobago, Paraguay, Brasil, and Mexico. On the public outreach areas, there were widespread activities during the International Year of Astronomy 2009, that have triggered interest in the communities to do follow up actions. About primary or secondary studies, although it is difficult to generalize, since each country has its own peculiarities; at least in Mexico, we have been unable to impact at this level.

To conclude, there are many aspects that can be pursued at all levels so there is plenty of work in this field. Given the large size of the continent, perhaps several OADs should be supported for specific regions, with target goals within the IAU program.

I am grateful to DGAPA-PAPIIT-IN105511 and CONACyT 129753 for their support. 